\title{
Axon Guidance Molecules Guiding Neuroinflammation
}

\author{
Won Suk Lee ${ }^{1,2}$, Won-Ha Lee ${ }^{3}$, Yong Chul Bae ${ }^{4}$ and Kyoungho Suk ${ }^{1,2 *}$ \\ ${ }^{1}$ Department of Pharmacology, School of Medicine, Kyungpook National University, Daegu 41944, ${ }^{2}$ Brain Science and \\ Engineering Institute, Kyungpook National University, Daegu 41944, ${ }^{3}$ BK21 Plus KNU Creative BioResearch Group, School of \\ Life Sciences, Kyungpook National University, Daegu 41566, ${ }^{4}$ Department of Anatomy and Neurobiology, School of Dentistry, \\ Kyungpook National University, Daegu 41940, Korea
}

Axon guidance molecules (AGMs), such as Netrins, Semaphorins, and Ephrins, have long been known to regulate axonal growth in the developing nervous system. Interestingly, the chemotactic properties of AGMs are also important in the postnatal period, such as in the regulation of immune and inflammatory responses. In particular, AGMs play pivotal roles in inflammation of the nervous system, by either stimulating or inhibiting inflammatory responses, depending on specific ligand-receptor combinations. Understanding such regulatory functions of AGMs in neuroinflammation may allow finding new molecular targets to treat neurodegenerative diseases, in which neuroinflammation underlies aetiology and progression.

Key words: Axon guidance molecule, Neuroinflammation, Glia, Neurodegenerative disease

\section{INTRODUCTION}

Axon guidance molecules (AGMs) are involved in several developmental processes in which determining directionality is important, such as body axis formation, neuronal migration, and axonal growth [1]. However, recent studies have revealed that AGMs are also involved in immune and inflammatory responses in peripheral organs/tissues and the central nervous system (CNS), postnatally [2-4]. In particular, there is evidence that different combinations of AGM ligand-receptor interactions are involved in neuroinflammation [5-7], which results from a series of immunological and inflammatory processes occurring within the nervous

Received March 27,2019, Revised May 8, 2019,

Accepted May 9, 2019

* To whom correspondence should be addressed. TEL: 82-53-420-4835, FAX: 82-53-256-1566 e-mail:ksuk@knu.ac.kr system. Various pro-inflammatory stimuli activate those processes, including pathogenic insults such as viral or bacterial infections, autoimmune responses, traumatic injuries, and proteinopathies. Neuroinflammation is thought to be of critical importance to degenerative brain diseases such as Alzheimer's disease, Parkinson's disease, amyotrophic lateral sclerosis, and multiple sclerosis [8-10]. Recently, multiple studies tried to elucidate the precise mechanisms underlying neuroinflammation and the resulting neurodegenerative diseases and to identify relevant therapeutic solutions. However, how neuroinflammation and associated neurodegenerative disorders are triggered and regulated remains poorly understood.

Axon guidance is the neurodevelopmental process whereby axons find the correct direction of growth across the developing nervous system to reach appropriate targets for their neurons [11]. The main functions of neurons are receiving, producing, relaying, integrating, and saving information. Axon guidance contributes to the construction and setting up of the 'infrastructure' that allows 
the nervous system to function. For a given neuron, axon guidance begins when, among a number of other neurites that become dendrites, one protrudes further from the cell body to give rise to the pioneer axon [12]. Earlier growth of glial cells, which produce the "slings", is essential for pioneer axons to find their appropriate targets [11]. To regulate this process, glial cells and axons communicate through the molecular interactions between axon guidance cues and receptors [1]. Axon guidance cues are present on the cell surface of and/or secreted by cells neighboring the protruding axon such as glial cells, muscle cells, ependymal cells, or other neurons. For example, Netrin is secreted to the extracellular matrix (ECM) from the ventral glia and neurons in C. elegans, and from the CNS midline glia and neurons in Drosophila and mammals, to regulate the directional guidance of the axons that later form the midline commissures $[13,14]$. Some ECM proteins also work as guidance cues. Depending upon the localization, they exert a combination of 'permissive' role which allows the outgrowth of axons, and 'instructive' function to mediate the directional guidance of axons [15]. Such permissive and instructive functions of the AGMs on axon outgrowth also determine the chemotactic cellular behaviors in postnatal biological processes such as angiogenesis [16], axonal regeneration [17], and inflammatory responses [18]. Therefore, understanding of the chemotactic functions of AGMs in development may allow the prediction of some of their postnatal functions; indeed, many AGMs are known to possess immunomodulatory functions [2].

Overall, immune and inflammatory responses start with the recognition of pro-inflammatory stimuli such as infection or tissue damage. Next, immune and inflammatory cells are activated by increased cytokine secretion and cell migration. Finally, the resulting edema and scavenging activities lead to resolution and repair [19]. A number of recent studies have revealed that AGMs are involved in most of these steps of the inflammatory process, including in the triggering and resolving of inflammation [2]. In general, proinflammatory cytokines are involved in the chemoattraction of immune and inflammatory cells such as macrophages and neutrophils. However, AGMs exhibit both attractive and repulsive chemotactic functions [20]. Depending upon the combinations of the ligand-receptor pairs present, AGMs can attract or repulse cells, and thus can be involved in both the stimulation and resolution of inflammatory responses [21-23]. The roles of AGMs within the nervous system are well known, including in axon guidance, synaptogenesis, neuronal migration, axon regeneration after injury, and regulation of neuroinflammation. In this review, we discuss the important roles of AGMs in the inflammation of the nervous system.

\section{AXON GUIDANCE MOLECULES IN NEUROINFLAMMATION}

Throughout the process of neuroinflammation, glial cells play central roles in initiating and sustaining immune and inflammatory responses. Glial cells produce AGMs even after completion of CNS development [24], and there is evidence that the expression levels of AGMs are changed by inflammatory stimuli in the nervous system $[25,26]$. At the same time, such changes contribute to the regulation of neuroinflammation, from onset to resolution. Many AGMs become upregulated by pro-inflammatory processes such as pathogenic infections [27], physical or ischemic injuries [5], or by pathological conditions such as beta-amyloid accumulation in Alzheimer's disease [28]. In these cases, AGMs play either protective or detrimental roles, depending upon their receptor-ligand combinations (Table 1).

\section{Netrin pathway in neuroinflammation}

Netrins are a secreted type of AGM ligands mostly involved in mid- to long-range guidance, including in the midline crossing of commissural axons [29]. However, Netrin function is not limited to the developing nervous system. Recent studies showed that $\mathrm{Ne}$ trins can act postnatally as well, in any process in which chemotactic cellular activation is important, especially immune responses [30]. Curiously, the fact that Netrin-mediated chemotactic regulation occurs in non-neuronal systems ultimately renews our interest in their roles in the nervous system, albeit in particular after the completion of development.

It is well known that Netrins exhibit attractive or repulsive bifunctionality in regulating directional guidance, depending upon the types of receptors they interact with: Deleted in Colorectal Cancer (DCC) for attraction, and UNC5 homodimers or heterodimers with DCC, for repulsion [1]. Recently, the repulsive chemotactic activity of UNC5 receptors has drawn much attention in the fields of neuroscience and neurology, because it is suggested to have inhibitory roles in inflammatory responses in the nervous system, and thus to have neuroprotective roles in neurodegenerative diseases [18, 31].

Related to their neuroprotective roles, Netrins also appear to exhibit anti-inflammatory activity; a number of genetic, molecular and biochemical studies has revealed that Netrin signaling activates anti-inflammatory, or neuroprotective, signaling pathways in neurological disease models, where neuroinflammation contributes either to the onset of the disease, or a detrimental prognosis $[5,7,25,32]$. In primary cultured human endothelial cells, Netrin expression is upregulated by stimulation with TNF- $\alpha$ and IFN- $\gamma$ [4]. These results suggest that Netrin signaling is activated by pro-inflammatory stimulation, and therefore that Netrin may 
Table 1. Summary of the roles of AGMs in neuroinflammation described in this review

\begin{tabular}{|c|c|c|c|c|c|}
\hline Ligands & Source & Receptors & Target & Role in neuroinflammation & $\begin{array}{l}\text { Refer- } \\
\text { ences }\end{array}$ \\
\hline \multirow[t]{3}{*}{ Netrin-1 } & Recombinant & APP & Neuron & Inhibits amyloid- $\beta$ production & {$[33]$} \\
\hline & Endothelial cell & UNC5B & Microglia \& Astrocyte & Downregulates astrocytic \& microglial activation & {$[5,32]$} \\
\hline & Recombinant & $\begin{array}{l}\text { DCC/APP } \\
\text { complex }\end{array}$ & Recombinant & Blocks $\gamma$-secretase cleavage & {$[34]$} \\
\hline Sema $3 \mathrm{~A}$ & PBMC & ND & - & Anti-inflammatory function in multiple sclerosis model & {$[43,44]$} \\
\hline ND & - & Sema 4B & Astrocyte & Induces astrogliosis & {$[41]$} \\
\hline Sema 4D & Recombinant & $\begin{array}{l}\text { PlexinB1, } \\
\text { CD72 }\end{array}$ & Microglia & Inhibits LPS-stimulated microglial activation & {$[26]$} \\
\hline Sema 7A & Purkinje neuron & PlexinC1 & Purkinje neuron & Required for LPS-induced neuroinflammation & {$[45]$} \\
\hline EphrinA1 & Endothelial cell & EphA2 & Endothelial cell & Mediates neuroinflammation by pathogens & {$[52]$} \\
\hline $\begin{array}{l}\text { EphrinA1 } \\
\text { \&A3 }\end{array}$ & Neuron \& Astrocyte & EphA2 & $\begin{array}{l}\text { Neuron \& Endothelial } \\
\text { cell }\end{array}$ & Mediates neuroinflammation by ischemia & {$[53]$} \\
\hline ND & - & EphA4 & Astrocyte & Required for astrocyte migration & {$[55]$} \\
\hline EphB1 & Motoneuron & EphrinB1 & Astrocyte & Retrograde signal for astrocyte $\mathrm{A} 2$ polarization & {$[56]$} \\
\hline \multirow{2}{*}{ Slit2 } & Neuron \& Astrocyte & Robol & Peripheral immune cell & Inhibits peripheral immune cell infiltration & {$[61]$} \\
\hline & Recombinant & Robo4 & Endothelial cell & Protects blood-brain barrier in brain injury & {$[6]$} \\
\hline Wnt3a & Glioblastoma & ND & - & Stimulates M2-like microglial activation in glioblastoma & {$[67]$} \\
\hline Wnt5a & Microglia & ND & - & With Wnt3a, inhibits LPS-stimulated microglial activation & {$[68]$} \\
\hline
\end{tabular}

$\mathrm{ND}$, not determined with respect to the role of AGM in regulating neuroinflammation; PBMC, peripheral blood mononuclear cells.

be involved in cellular reactions triggered by neuroinflammation [7]. Similarly, treatment with recombinant Netrin-1 can achieve anti-inflammatory responses through activation of the microglial UNC5B/PPAR $\gamma / N F-\kappa B$ signaling pathway [5], and astrocytic pAKT and PPAR $\gamma$ activation through the same UNC5B receptor [32].

Netrin-1 contributes to reducing neuroinflammation in the brain by blocking the gamma-secretase processing of amyloid precursor proteins (APP) and thus decreasing the production of betaamyloid [33]. As one of the main pathogenic consequences of the accumulation of beta-amyloid is neuroinflammation, Netrin's role in neuroprotection may come from non-cell-autonomous activities. The Netrin receptor DCC forms a complex with APP and produces alpha-secretase-processed sAPPa [34], and this complex reinforces Netrin-mediated axon guidance signals, such as the DCC-mediated activation of ERK1/2 kinases [35]. In pathological conditions, APP undergoes gamma-secretase processing to produce beta-amyloid, and in this case, Netrin-1 negatively regulates the cleavage activity to reduce the levels of beta-amyloid [33]. In addition to regulating post-translational processing, Netrin-1 also decreases intracellular neurotoxic signals triggered by beta-amyloid (1-42) fragments [36]. In human subjects, a mutation in the Netrin receptor UNC5C was found to be correlated to the autosomal dominant type of late-onset Alzheimer's disease, and expressing that mutation in neurons causes increased neuronal cell death in the hippocampus [37]. Such observations indicate that Netrins are key players in the resolution of neuroinflammation, and that
Netrin signaling components may be promising candidates as therapeutic targets against neurological diseases.

\section{Semaphorin pathway in neuroinflammation}

Semaphorins and their receptors (Neuropilins and Plexins) are AGMs mostly involved in contact repulsion during neural development [38]. Semaphorins are members of a large protein family, which consists of eight sub-families with at least thirty members. Among them, in vertebrates, there are twenty members in five evolutionary conserved sub-families (Class 3 to 7 Semaphorins). They exist in transmembrane, secreted, or glycosylphosphatidylinositol (GPI)-linked forms, and are involved in diverse cell-to-cell communication processes that require cytoskeletal modification and cell adhesion or migration. Some Semaphorins (e.g., 3A, 4A, and $4 \mathrm{D})$ play important roles in immune responses and are defined as "immune Semaphorins [39]."

It is well known that Semaphorins are involved in the onset and the resolution of neuroinflammation, but the precise regulatory mechanisms underlying cell-to-cell or intracellular interactions are yet to be understood. Therefore, efforts to prevent neurodegenerative diseases have been targeting "immune Semaphorins" [40]. They have pivotal roles in regulating neuroinflammatory responses, with their specific role depending upon the ligandreceptor combinations present. Semaphorin 4D mitigates proinflammatory microglial activation in response to the bacterial endotoxin lipopolysaccharide (LPS) [26]. In the zebrafish spinal cord injury model, activated microglia are recruited to neurons ex- 
pressing Semaphorin 4D, and inhibiting Semaphorin 4D hinders locomotive recovery and axon regeneration [17]. For astrocyte activation, Semaphorin $4 \mathrm{~B}$ functions as the receptor for activation of astrogliosis followed by brain injury [41]. The only secreted Semaphorins that are involved in the immune responses are the Class 3 Semaphorins [42]. Semaphorin 3A is one of them, regulating the neuroinflammatory responses in multiple sclerosis (MS) models [43]. In MS patients, the expression levels of Semaphorin $3 \mathrm{~A}$ were significantly decreased, suggesting its inhibitory role in the immunopathogenesis of MS [44]. The membrane-anchored Semaphorin 7A is also involved in neuroinflammation, as it is required for LPS-stimulated demyelination, or cell death, in both in vitro cerebellar slice cultures and in vivo experimental autoimmune encephalomyelitis (EAE) models [45].

\section{Ephrin pathway in neuroinflammation}

Erythropoietin-producing human hepatocellular receptors (Ephs) form a major axon guidance receptor protein family, which has the membrane-bound Ephrins as the corresponding family of ligands [46]. The Eph receptors comprise the largest known receptor tyrosine kinase family; it consists of fourteen members, including nine EphA and five EphB receptor subtypes [47]. Depending on their main target Ephs, Ephrin ligands can also be subcategorized into A- and B-types [48]. Ephrin-As are glycosylphosphatidylinositol (GPI)-anchored extracellular proteins, whereas Ephrin-Bs are transmembrane proteins. The main role of Ephrins and their Eph receptors is in contact-mediated repulsion during axon outgrowth in neural development [49]. Ephrin signals are bi-directional in terms of cell-to-cell interactions, therefore both Ephs and Ephrins are able to relay signals for each other, especially in the B-type subfamily; both EphBs and Ephrin-Bs have intracellular tyrosine residues that need to be phosphorylated to transmit extracellular signals [50].

Other than in axon guidance, Ephrin/Eph signaling plays important roles in immune activities [50]. For example, EphA2 mediates inflammatory responses in the post-infectious irritable bowel syndrome (PI-IBS) model [51]. Chemically inhibiting EphA2 mitigates pro-inflammatory responses in PI-IBS model mice. Some Eph receptors also function in the detection of pathogen activities. For example, EphA2 receptors detect fungal beta-glucan in epithelial cells [27]. In blood-brain barrier endothelial cells, EphA2 internalizes Cryptococcus neoformans, a yeast pathogen which causes meningitis [52]. Combined with the finding that EphA2 causes neuronal death after ischemic brain injury [53], it has been suggested that EphA2, along with its ligands (Ephrins A1 A3), are involved in the activation of pro-inflammatory responses against pathogens.
In the nervous system, Ephrins and Eph receptors relay bidirectional signals between neurons and glial cells to regulate inflammatory activation of glia [54]. Both ligands and receptors are expressed in neurons and astrocytes of adult brains [24], and are involved in the activation of inflammatory signals between glia and neurons. Eph receptors are known for their role in reactive astrogliosis. For example, blocking EphA4 activity by specific chemical inhibitors reduces astrocyte migratory activation in the in vitro model of scratch injury [55]. As for retrograde signals, in a post-axotomy model, astrocytic EphrinB1 receives signals from neuronal EphB1 to activate a neuroprotective phenotype (namely A2-like activation) through the phosphorylation of STAT3 [56]. This signaling is hindered in SOD-1 mutant, human induced pluripotent stem cell-derived astrocytes, an in vitro disease model for amyotrophic lateral sclerosis. These observations imply that a proinflammatory bias may take place upon inflammatory glial activation, and thus resolving the neuroinflammation to a resting phase becomes difficult under the disease condition.

\section{OTHER AXON GUIDANCE MOLECULES INVOLVED IN NEU- ROINFLAMMATION}

Besides those mentioned above, other important evolutionarily conserved AGMs include Slit/Robo and Wnt/Fz. In addition to roles in guiding axons, they are also involved in various chemotaxis-dependent cellular processes, including cell migration and immune cell activation during neuroinflammatory responses [2].

\section{Slit pathway in neuroinflammation}

Slit proteins are AGMs also known to function in inflammation. One interesting property of the Slit ligands is that they can interact with different receptors by undergoing proteolytic processing [57]. For example, Slit2 is cleaved into N-terminal and C-terminal fragments, which differentially regulate the chemotactic behaviors of neutrophils via attractive and repulsive effects, respectively [58]. In the nervous system, the N-terminal Slit2 fragment interacts with APPs [59], the precursor of the beta-amyloid peptide which contributes to Alzheimer's disease. Meanwhile, the C-terminal Slit2 fragment mediates growth cone collapse by interacting with Plexin A1 [60], which normally function as a Semaphorin receptor. Such redundancy and overlaps in ligand-receptor interactions add complexity to the Slit-mediated guidance signals. This may allow diversity in signals available for triggering by multiple specific contexts.

One important function of the Slit pathway is to regulate neuroinflammatory responses. Most studies on the inflammatory responses of the Slit pathway focused on Slit2 [58], and this also 
seems to be the case in the nervous system. Slit2 mitigates neuroinflammation using redundancy in ligand-receptor interactions. Slit2 protects the blood-brain barrier by interacting with Robo4 in surgical brain injury [6], while it inhibits infiltration of peripheral immune cells through Robol [61]. However, whether the different cleaved forms of Slits have different functions in neuroinflammatory responses is not known. Therefore, overexpression of Slit2 may be a way to improve the prognosis of patients with neuroinflammation-dependent neurological conditions [62]. However, direct application of Slit2 should be tested with care, because each variant isotype of the protein could have a different effect [58].

\section{Wnt pathway in neuroinflammation}

Wnts are important signaling proteins functioning in developmental and several other cellular processes, including axis formation, cell fate determination, cell migration, survival, and proliferation [63]. During development of the nervous system, Wnts function as important guidance cues in determining cell polarity, axon growth cone directionality, and neuron cell body orientation [64]. Postnatally, Wnts are also involved in neuroinflammatory responses [65].

Wnt signaling through beta-catenin is the main process that controls the activation spectrums of immunoreactive gliosis: either to a neurodegenerative M1-like or a neuroprotective M2-like phenotype [66]. Wnt3a, secreted from glioblastoma cells, stimulates the activation of the microglia neuroprotective M2-like phenotype by activating beta-catenin signaling [67]. This represents the immu- nosuppressive role of the canonical Wnt signal on microglial cells, which is shown by the characteristic activity of glioma cells in the brain. Such activity of Wnt3a seems to depend on the type of stimuli received and acts together with Wnt5a to determine whether pro- or anti-inflammatory responses take place. Solely adding recombinant Wnt proteins activates pro-inflammatory responses in microglia, whereas if added together with LPS, recombinant Wnts alleviate the LPS-stimulated microglial inflammatory activation [68].

\section{GUIDING FOR THE FUTURE: AGMS AS POTENTIAL TARGETS TO CONTROL NEUROINFLAMMATION}

As we have discussed in this review, AGMs play pivotal roles in various aspects of chemotactic behavior, including in inflammatory responses of glia, in peripheral immune responses, and in axon pathfinding. The bi-directionality of their function can be observed in multiple types of tissues, and in neuroinflammation, it enables AGMs to "guide" the process from onset to resolution. The combination of ligand-receptor interactions is key for determining the AGM-guided polarity of immune responses (Fig. 1). Importantly, this characteristic of AGMs makes them excellent therapeutic targets for promoting neuroprotection against proinflammatory insults, while alleviating neurodegeneration.

Understanding of how the molecular mechanisms underlying AGM regulation of neuroinflammation link together is still poor, because of a lack of comprehensive studies integrating temporal

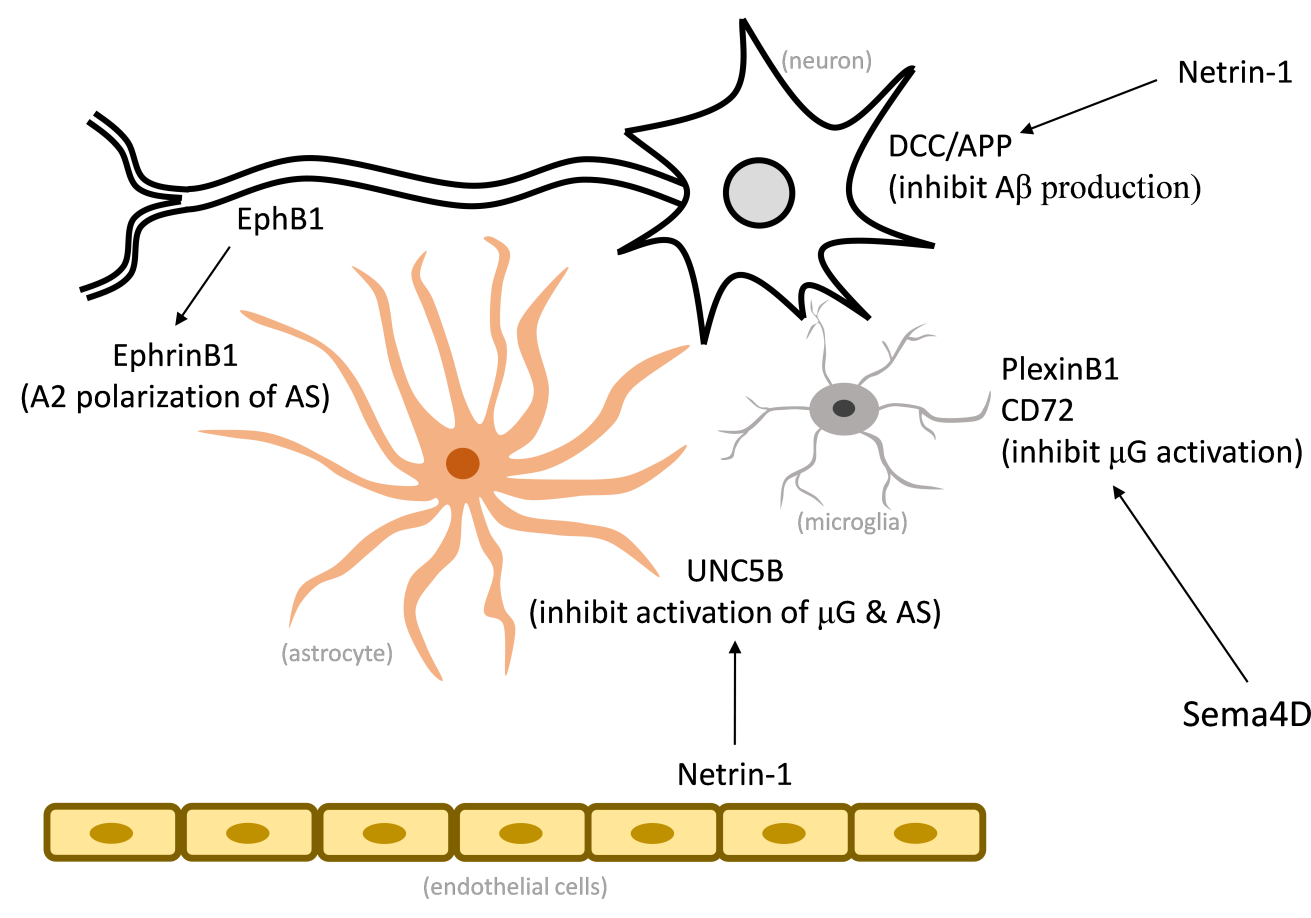

Fig. 1. Selected AGMs regulating neuroinflammation and neuron-glia interactions. Recombinant Netrin-1 inhibits $\mathrm{A} \beta$ production by interacting with APP and DCC receptors. Recombinant Sema4D inhibits microglial activation through microglial receptors PlexinB1 and CD72. UNC5B can inhibit inflammatory activation of microglia and astrocytes when bound to endothelial Netrin-1. Interaction between neuronal EphB1 and astrocyte EphrinB1 mediates neuroprotective activation of astrocytes. $A \beta$, amyloid beta; $\mu \mathrm{G}$, microglia; $\mathrm{AS}$, astrocyte. 
and spatial roles of AGMs in neuroinflammation. Another relevant research topic may be the AGMs' interactions with metalloproteinases and secretases. Metalloproteinases are involved in ectodomain shedding of receptor molecules, including the receptors of AGMs [33]. There are reports that Netrin and Slit receptors interact with metalloproteinases. These studies may provide insight into how to link those interactions with the onset of neurodegenerative diseases $[59,69]$. However, an exhaustive list-up of their combinatorial interactions is still lacking, and generating them would comprise a useful starting point to investigate these relationships.

As overexpression of AGM genes and treatment with recombinant AGM proteins modulate neuroinflammatory responses in neurodegenerative disease models, therapeutic applications could emerge that use these AGM targets. Some of the findings regarding the regulatory functions of AGMs in neuroinflammation became intellectual properties in the diagnosis, prevention, and treatment of related neurological diseases [70, 71]. These methods apply the anti-inflammatory or neuroprotective functions of AGMs, such as Netrin-1 and neuregulin-1, or inhibit the pro-inflammatory functions of other AGMs, such as Semaphorin 4D, to treat pathological conditions in Alzheimer's and Parkinson's diseases, for example. Most of these approaches focus on the role of AGMs in mitigating causative factors such as beta-amyloid, or in detecting AGMs as disease biomarkers, which together makes AGMs very promising targets. Still, these therapeutic and diagnostic efforts are mostly focused on the role of AGMs in neurons, rather than their roles in regulating neuroinflammatory responses [72], which became prominent as causative factors in the onset of neurodegenerative diseases. Therefore, there is great scope for further translational investigation of the roles of AGMs in neuroinflammation.

\section{ACKNOWLEDGEMENTS}

This work was supported by Basic Science Research Program through the National Research Foundation of Korea (NRF), which is funded by the Korean government (MSIT; grant nos. 2018R1A2A1A05077118, 2016M3C7A1904148, and NRF2017R1A5A2015391).

\section{REFERENCES}

1. Tessier-Lavigne M, Goodman CS (1996) The molecular biology of axon guidance. Science 274:1123-1133.

2. Mirakaj V, Rosenberger P (2017) Immunomodulatory functions of neuronal guidance proteins. Trends Immunol $38: 444-456$
3. Rosenberger P, Schwab JM, Mirakaj V, Masekowsky E, Mager A, Morote-Garcia JC, Unertl K, Eltzschig HK (2009) Hypoxia-inducible factor-dependent induction of netrin-1 dampens inflammation caused by hypoxia. Nat Immunol 10:195202.

4. Podjaski C, Alvarez JI, Bourbonniere L, Larouche S, Terouz S, Bin JM, Lécuyer MA, Saint-Laurent O, Larochelle C, Darlington PJ, Arbour N, Antel JP, Kennedy TE, Prat A (2015) Netrin 1 regulates blood-brain barrier function and neuroinflammation. Brain 138:1598-1612.

5. Xie Z, Huang L, Enkhjargal B, Reis C, Wan W, Tang J, Cheng Y, Zhang JH (2018) Recombinant netrin-1 binding UNC5B receptor attenuates neuroinflammation and brain injury via PPAR $\gamma / N F \kappa B$ signaling pathway after subarachnoid hemorrhage in rats. Brain Behav Immun 69:190-202.

6. Sherchan P, Huang L, Akyol O, Reis C, Tang J, Zhang JH (2017) Recombinant Slit2 reduces surgical brain injury induced blood brain barrier disruption via Robo4 dependent Racl activation in a rodent model. Sci Rep 7:746.

7. Mulero P, Córdova C, Hernández M, Martín R, Gutiérrez B, Muñoz JC, Redondo N, Gallardo I, Téllez N, Nieto ML (2017) Netrin-1 and multiple sclerosis: a new biomarker for neuroinflammation? Eur J Neurol 24:1108-1115.

8. Carson MJ, Thrash JC, Walter B (2006) The cellular response in neuroinflammation: The role of leukocytes, microglia and astrocytes in neuronal death and survival. Clin Neurosci Res 6:237-245.

9. Paulson HL (1999) Protein fate in neurodegenerative proteinopathies: polyglutamine diseases join the (mis)fold. Am J Hum Genet 64:339-345.

10. Norden DM, Muccigrosso MM, Godbout JP (2015) Microglial priming and enhanced reactivity to secondary insult in aging, and traumatic CNS injury, and neurodegenerative disease. Neuropharmacology 96:29-41.

11. Silver J, Lorenz SE, Wahlsten D, Coughlin J (1982) Axonal guidance during development of the great cerebral commissures: descriptive and experimental studies, in vivo, on the role of preformed glial pathways. J Comp Neurol 210:10-29.

12. Dotti CG, Banker GA (1987) Experimentally induced alteration in the polarity of developing neurons. Nature 330:254256.

13. Mitchell KJ, Doyle JL, Serafini T, Kennedy TE, Tessier-Lavigne M, Goodman CS, Dickson BJ (1996) Genetic analysis of netrin genes in Drosophila: netrins guide CNS commissural axons and peripheral motor axons. Neuron 17:203-215.

14. Wadsworth WG, Bhatt H, Hedgecock EM (1996) Neuroglia and pioneer neurons express UNC-6 to provide global and 
local netrin cues for guiding migrations in C. elegans. Neuron 16:35-46.

15. Yang Y, Lee WS, Tang X, Wadsworth WG (2014) Extracellular matrix regulates UNC-6 (netrin) axon guidance by controlling the direction of intracellular UNC-40 (DCC) outgrowth activity. PLoS One 9:e97258.

16. Li Y, Liu DX, Li MY, Qin XX, Fang WG, Zhao WD, Chen YH (2014) Ephrin-A3 and ephrin-A4 contribute to microgliainduced angiogenesis in brain endothelial cells. Anat Rec (Hoboken) 297:1908-1918.

17. Peng SX, Yao L, Cui C, Zhao HD, Liu CJ, Li YH, Wang LF, Huang SB, Shen YQ (2017) Semaphorin4D promotes axon regrowth and swimming ability during recovery following zebrafish spinal cord injury. Neuroscience 351:36-46.

18. Paradisi A, Mehlen P (2010) Netrin-1, a missing link between chronic inflammation and tumor progression. Cell Cycle 9:1253-1262.

19. Sugimoto MA, Sousa LP, Pinho V, Perretti M, Teixeira MM (2016) Resolution of inflammation: what controls its onset? Front Immunol 7:160.

20. Santiago-Lopez AJ (2018) A repulsive environment induces neurodegeneration of midbrain dopaminergic neurons. J Neurosci 38:1323-1325.

21. Ranganathan PV, Jayakumar C, Mohamed R, Dong Z, Ramesh G (2013) Netrin-1 regulates the inflammatory response of neutrophils and macrophages, and suppresses ischemic acute kidney injury by inhibiting COX-2-mediated PGE2 production. Kidney Int 83:1087-1098.

22. Shimizu T, Smits R, Ikenaka K (2016) Microglia-induced activation of non-canonical Wnt signaling aggravates neurodegeneration in demyelinating disorders. Mol Cell Biol 36:2728-2741.

23. Mediero A, Ramkhelawon B, Wilder T, Purdue PE, Goldring SR, Dewan MZ, Loomis C, Moore KJ, Cronstein BN (2016) Netrin-1 is highly expressed and required in inflammatory infiltrates in wear particle-induced osteolysis. Ann Rheum Dis 75:1706-1713.

24. Jha MK, Kim JH, Song GJ, Lee WH, Lee IK, Lee HW, An SS, Kim S, Suk K (2018) Functional dissection of astrocytesecreted proteins: implications in brain health and diseases. Prog Neurobiol 162:37-69.

25. Moon C, Kim H, Ahn M, Jin JK, Wang H, Matsumoto Y, Shin $\mathrm{T}$ (2006) Enhanced expression of netrin-1 protein in the sciatic nerves of Lewis rats with experimental autoimmune neuritis: possible role of the netrin-1/DCC binding pathway in an autoimmune PNS disorder. J Neuroimmunol 172:6672.
26. Toguchi M, Gonzalez D, Furukawa S, Inagaki S (2009) Involvement of Sema4D in the control of microglia activation. Neurochem Int 55:573-580.

27. Swidergall M, Solis NV, Lionakis MS, Filler SG (2018) EphA2 is an epithelial cell pattern recognition receptor for fungal $\beta$-glucans. Nat Microbiol 3:53-61.

28. Vargas LM, Cerpa W, Muñoz FJ, Zanlungo S, Alvarez AR (2018) Amyloid- $\beta$ oligomers synaptotoxicity: The emerging role of EphA4/c-Abl signaling in Alzheimer's disease. Biochim Biophys Acta Mol Basis Dis 1864:1148-1159.

29. Van Battum EY, Brignani S, Pasterkamp RJ (2015) Axon guidance proteins in neurological disorders. Lancet Neurol 14:532-546.

30. Feinstein J, Ramkhelawon B (2017) Netrins \& semaphorins: novel regulators of the immune response. Biochim Biophys Acta Mol Basis Dis 1863:3183-3189.

31. Li Q, Wang BL, Sun FR, Li JQ, Cao XP, Tan L (2018) The role of UNC5C in Alzheimer's disease. Ann Transl Med 6:178.

32. He X, Liu Y, Lin X, Yuan F, Long D, Zhang Z, Wang Y, Xuan A, Yang GY (2018) Netrin-1 attenuates brain injury after middle cerebral artery occlusion via downregulation of astrocyte activation in mice. J Neuroinflammation 15:268.

33. Spilman PR, Corset V, Gorostiza O, Poksay KS, Galvan V, Zhang J, Rao R, Peters-Libeu C, Vincelette J, McGeehan A, Dvorak-Ewell M, Beyer J, Campagna J, Bankiewicz K, Mehlen P, John V, Bredesen DE (2016) Netrin-1 interrupts amyloid- $\beta$ amplification, increases sA $\beta P P \alpha$ in vitro and in vivo, and improves cognition in a mouse model of Alzheimer's disease. J Alzheimers Dis 52:223-242.

34. Borel F, Marzocca F, Delcros JG, Rama N, Mehlen P, Ferrer JL (2017) Molecular characterization of netrin-1 and APP receptor binding: new leads to block the progression of senile plaques in Alzheimer's disease. Biochem Biophys Res Commun 488:466-470.

35. Rama N, Goldschneider D, Corset V, Lambert J, Pays L, Mehlen P (2012) Amyloid precursor protein regulates netrin1-mediated commissural axon outgrowth. J Biol Chem 287:30014-30023.

36. Zamani E, Parviz M, Roghani M, Mohseni-moghaddam P (2018) Key mechanisms underlying netrin-1 prevention of impaired spatial and object memory in $A \beta_{1-42}$ CA1-injected rats. Clin Exp Pharmacol Physiol 46:86-93.

37. Wetzel-Smith MK, Hunkapiller J, Bhangale TR, Srinivasan K, Maloney JA, Atwal JK, Sa SM, Yaylaoglu MB, Foreman O, Ortmann W, Rathore N, Hansen DV, Tessier-Lavigne M, Mayeux R, Pericak-Vance M, Haines J, Farrer LA, Schellenberg GD, Goate A, Behrens TW, Cruchaga C, Watts RJ, Graham RR 
(2014) A rare mutation in UNC5C predisposes to late-onset Alzheimer's disease and increases neuronal cell death. Nat Med 20:1452-1457.

38. Alto LT, Terman JR (2017) Semaphorins and their signaling mechanisms. Methods Mol Biol 1493:1-25.

39. Vadasz Z, Toubi E (2014) Semaphorins: their dual role in regulating immune-mediated diseases. Clin Rev Allergy Immunol 47:17-25.

40. LaGanke C, Samkoff L, Edwards K, Jung Henson L, Repovic P, Lynch S, Stone L, Mattson D, Galluzzi A, Fisher TL, Reilly C, Winter LA, Leonard JE, Zauderer M (2017) Safety/tolerability of the anti-semaphorin 4D Antibody VX15/2503 in a randomized phase 1 trial. Neurol Neuroimmunol Neuroinflamm 4:e367.

41. Ben-Gigi L, Sweetat S, Besser E, Fellig Y, Wiederhold T, Polakiewicz RD, Behar O (2015) Astrogliosis induced by brain injury is regulated by Sema4B phosphorylation. eNeuro 2:ENEURO.0078-14.2015.

42. Tian X, Gan H, Zeng Y, Zhao H, Tang R, Xia Y (2018) Inhibition of semaphorin-3a suppresses lipopolysaccharideinduced acute kidney injury. J Mol Med (Berl) 96:713-724.

43. Eixarch H, Gutiérrez-Franco A, Montalban X, Espejo C (2013) Semaphorins 3A and 7A: potential immune and neuroregenerative targets in multiple sclerosis. Trends Mol Med 19:157164.

44. Rezaeepoor M, Shapoori S, Ganjalikhani-Hakemi M, Etemadifar M, Alsahebfosoul F, Eskandari N, Mansourian M (2017) Decreased expression of Sema3A, an immune modulator, in blood sample of multiple sclerosis patients. Gene 610:59-63.

45. Gutiérrez-Franco A, Eixarch H, Costa C, Gil V, Castillo M, Calvo-Barreiro L, Montalban X, Del Río JA, Espejo C (2017) Semaphorin $7 \mathrm{~A}$ as a potential therapeutic target for multiple sclerosis. Mol Neurobiol 54:4820-4831.

46. Attwood BK, Patel S, Pawlak R (2012) Ephs and ephrins: emerging therapeutic targets in neuropathology. Int J Biochem Cell Biol 44:578-581.

47. Taylor H, Campbell J, Nobes CD (2017) Ephs and ephrins. Curr Biol 27:R90-R95.

48. Coulthard MG, Morgan M, Woodruff TM, Arumugam TV, Taylor SM, Carpenter TC, Lackmann M, Boyd AW (2012) Eph/Ephrin signaling in injury and inflammation. Am J Pathol 181:1493-1503.

49. Hattori M, Osterfield M, Flanagan JG (2000) Regulated cleavage of a contact-mediated axon repellent. Science 289:13601365.

50. Pasquale EB (2008) Eph-ephrin bidirectional signaling in physiology and disease. Cell 133:38-52.

51. Zeng L, Li K, Wei H, Hu J, Jiao L, Yu S, Xiong Y (2018) A novel EphA2 inhibitor exerts beneficial effects in PI-IBS in vivo and in vitro models via $\mathrm{Nrf} 2$ and $\mathrm{NF}-\kappa \mathrm{B}$ signaling pathways. Front Pharmacol 9:272.

52. Aaron PA, Jamklang M, Uhrig JP, Gelli A (2018) The bloodbrain barrier internalises Cryptococcus neoformans via the EphA2-tyrosine kinase receptor. Cell Microbiol 20:e12811.

53. Thundyil J, Manzanero S, Pavlovski D, Cully TR, Lok KZ, Widiapradja A, Chunduri P, Jo DG, Naruse C, Asano M, Launikonis BS, Sobey CG, Coulthard MG, Arumugam TV (2013) Evidence that the EphA2 receptor exacerbates ischemic brain injury. PLoS One 8:e53528.

54. Cissé M, Checler F (2015) Eph receptors: new players in Alzheimer's disease pathogenesis. Neurobiol Dis 73:137-149.

55. Parmentier-Batteur S, Finger EN, Krishnan R, Rajapakse HA, Sanders JM, Kandpal G, Zhu H, Moore KP, Regan CP, Sharma S, Hess JF, Williams TM, Reynolds IJ, Vacca JP, Mark RJ, Nantermet PG (2011) Attenuation of scratch-induced reactive astrogliosis by novel EphA4 kinase inhibitors. J Neurochem 118:1016-1031.

56. Tyzack GE, Hall CE, Sibley CR, Cymes T, Forostyak S, Carlino G, Meyer IF, Schiavo G, Zhang SC, Gibbons GM, Newcombe J, Patani R, Lakatos A (2017) A neuroprotective astrocyte state is induced by neuronal signal EphB1 but fails in ALS models. Nat Commun 8:1164.

57. Bellon A, Mann F (2018) Keeping up with advances in axon guidance. Curr Opin Neurobiol 53:183-191.

58. Pilling D, Chinea LE, Consalvo KM, Gomer RH (2019) Different isoforms of the neuronal guidance molecule Slit2 directly cause chemoattraction or chemorepulsion of human neutrophils. J Immunol 202:239-248.

59. Wang B, Li H, Mutlu SA, Bowser DA, Moore MJ, Wang MC, Zheng H (2017) The amyloid precursor protein is a conserved receptor for Slit to mediate axon guidance. eNeuro 4:ENEURO.0185-17.2017.

60. Schiweck J, Beauchamp M, Humo M, Lelievre V (2015) Old friends, new story: the role of Slit2C signaling through PlexinA1. Cell Adhes Migr 9:417-421.

61. Sherchan P, Huang L, Wang Y, Akyol O, Tang J, Zhang JH (2016) Recombinant Slit2 attenuates neuroinflammation after surgical brain injury by inhibiting peripheral immune cell infiltration via Robol-srGAP1 pathway in a rat model. Neurobiol Dis 85:164-173.

62. Li G, He X, Li H, Wu Y, Guan Y, Liu S, Jia H, Li Y, Wang L, Huang R, Pei Z, Lan Y, Zhang Y (2018) Overexpression of Slit2 improves function of the paravascular pathway in the 
aging mouse brain. Int J Mol Med 42:1935-1944.

63. Willert K, Nusse R (2012) Wnt proteins. Cold Spring Harb Perspect Biol 4:a007864.

64. Zou Y (2004) Wnt signaling in axon guidance. Trends Neurosci 27:528-532.

65. Marchetti B, Pluchino S (2013) Wnt your brain be inflamed? Yes, it Wnt! Trends Mol Med 19:144-156.

66. L'Episcopo F, Tirolo C, Serapide MF, Caniglia S, Testa N, Leggio L, Vivarelli S, Iraci N, Pluchino S, Marchetti B (2018) Microglia polarization, gene-environment interactions and Wnt/ $\beta$-catenin signaling: emerging roles of glia-neuron and gliastem/neuroprogenitor crosstalk for dopaminergic neurorestoration in aged Parkinsonian brain. Front Aging Neurosci 10:12

67. Matias D, Dubois LG, Pontes B, Rosário L, Ferrer VP, BalçaSilva J, Fonseca AC, Macharia LW, Romão L, E Spohr TC, Chimelli L, Filho PN, Lopes MC, Abreu JG, Lima FR, MouraNeto V (2019) GBM-derived Wnt3a induces M2-like pheno- type in microglial cells through Wnt/ $\beta$-catenin signaling. Mol Neurobiol 56:1517-1530.

68. Halleskog C, Schulte G (2013) WNT-3A and WNT-5A counteract lipopolysaccharide-induced pro-inflammatory changes in mouse primary microglia. J Neurochem 125:803808.

69. Galko MJ, Tessier-Lavigne M (2000) Function of an axonal chemoattractant modulated by metalloprotease activity. Science 289:1365-1367.

70. Webster CI, Thom AG, Jermutus LU, Hatcher JP (2018) Blood brain barrier transport molecules and uses thereof. U.S. Pat. Application No. 15/536,371.

71. Smith ES, Zauderer M, Bowers WJ, Jonason A (2017) Use of semaphorin-4D binding molecules for treating neurodegenerative disorders. U.S. Pat. Application No. 9,598,495.

72. Jha MK, Lee WH, Suk K (2016) Functional polarization of neuroglia: implications in neuroinflammation and neurological disorders. Biochem Pharmacol 103:1-16. 